\title{
Pénfigo: Una revisión de la literatura
}

\section{Pemphigus: A literature review}

\author{
Matos-Cruz R*, Bascones-Martínez A**
}

\section{RESUMEN}

Dentro de las patologías que cursan con la formación de ampollas en la mucosa oral, existe un grupo importante de enfermedades mucocutáneas, entre las que se encuentra el pénfigo. El conocimiento de estas enfermedades tiene interés ya que son enfermedades que suelen iniciarse en la cavidad oral antes de expresarse en el resto del organismo, siendo que un diagnóstico y un tratamiento precoz mejoran mucho el pronóstico de estos pacientes. El pénfigo es una enfermedad ampollar poco frecuente, de etiología autoinmune, que afecta a la piel y mucosas. Constituye una entidad de carácter grave, curso agresivo y evolución crónica, que requiere tratamiento continuo y sistemático para evitar su evolución letal. De acuerdo a los criterios clínicos, histológicos e inmunológicos, se han reconocido cinco tipos de pénfigos: vulgar, vegetante, paraneoplásico, foliáceo y eritematoso. En el presente artículo se pretende realizar una revisión y puesta al día de esta enfermedad, centrándonos en las variantes que se expresan fundamentalmente con manifestaciones orales: pénfigo vulgar y pénfigo paraneoplásico. Se revisan los criterios etiopatológicos que nos llevan a comprender las manifestaciones clínicas, se analizan las formas de llegar al diagnóstico y los tratamientos que con mayor frecuencia se están utilizando en estos pacientes.

Palabras clave: Pénfigo, enfermedades vesículo-ampollares, clasificación, etiología, patogénesis, tratamiento.

\section{SUMMARY}

Within the pathologies that attend the formation of blisters in the oral mucosa there is an important group of mucocutaneous diseases, one of which is pemphigus. The knowledge of these diseases has been of such increasing interest because they usually begin in the oral cavity before expressing themselves in the rest of the organism, being that a diagnosis and a precocious treatment improve the prognosis of these patients. Pemphigus is a very uncommon bullous disease of autoimmune etiology that affects both the skin and the mucosa. It is a serious disease, with an aggressive course and of chronic evolution that requires continuous and systemic treatment in order to avoid its lethal evolution. According to the clinical, histological and immunological criteria, there are five types of pemphigus: vulgaris, vegetating, paraneoplastic, foliaceous and erythematous. The present article pretends to be an up-to-date review of this disease and centers on its variants that are essentially expressed as oral manifestations: pemphigus vulgaris and paraneoplastic pemphigus. We review the etiopathologic criteria which lead us to the understanding of its clinical manifestations and also analyze ways to get to a diagnosis and the most frequently used therapies in these patients.

Key words: Pemphigus, bullous diseases, classification, aetiology, pathogenesis, treatment

Fecha de recepción: 17 de septiembre de 2008.

Aceptado para publicación: 1 de octubre de 2008.

* Licenciada en Odontología. Alumna del Master en Periodoncia e Implantología UCM.

** Catedrático de Medicina Bucal y Periodoncia. Departamento de Estomatología III. Facultad de Odontología. Universidad Complutense de Madrid.

Matos-Cruz R, Bascones-Martínez A. Pénfigo: Una revisión de la literatura. Av. Odontoestomatol 2009; 25 (2): 67-82. 


\section{INTRODUCCIÓN}

Las enfermedades vesículo-ampollares son un grupo heterogéneo de enfermedades cuyo denominador común es la producción de vesículas o ampollas en la piel y mucosas (1).

Las enfermedades vesículo-ampollares que afectan a la mucosa oral constituyen un grupo importante de patologías de difícil diagnóstico. La distinción entre los varios tipos de lesiones, aunque estrictamente necesaria en el diagnóstico y orientación del tratamiento, no siempre es fácil (2). La fragilidad de las ampollas al traumatismo masticatorio y a la humedad hace con que sus techos se rompan rápidamente, determinando su vida corta como lesión clínica (2).

No obstante, es fundamental la identificación de las lesiones, bien como el conocimiento de las enfermedades. Esto permitirá orientar el diagnóstico e iniciar el tratamiento de forma correcta y precoz, lo que mejorará el pronóstico de estos pacientes y reducirá la morbilidad y mortalidad asociada a esta enfermedad (3).

Dentro de las patologías que cursan con la formación de ampollas en la mucosa oral, existe un grupo importante de enfermedades mucocutáneas, entre las que se encuentra el pénfigo. El conocimiento de estas enfermedades tiene interés ya que son enfermedades que suelen iniciarse en la cavidad oral antes de expresarse en el resto del organismo.

Bascones y cols. definen la lesión ampollosa como una lesión primaria, elevada y circunscrita, de más de $5 \mathrm{~mm}$ de diámetro, de contenido seroso o hemorrágico, base redondeada e irregular y de localización histológica a nivel intraepitelial o subepitelial (14). Este tipo de lesiones tiene una vida corta, dejando lesiones erosivas que pueden conservar restos del lecho ampollar. Pueden ubicarse en cualquier localización, aunque su prevalencia es mayor en las mucosas yugales de forma bilateral. Pueden ser pluriorificiales, de afectación general, el paciente tiene imposibilitada la masticación, deglución y fonación y pueden cursar adenopatías (14).

Grinspan dice que la distinción entre ampolla y vesícula no siempre es fácil (2). La ampolla tiene una sola cavidad (unilocular) y vida corta, ya que al punzar con un alfiler se vacía todo su contenido, se deseca y se concreta en una crosta (2). Puede ubicarse en cualquier localización, aunque su prevalencia es mayor en las mucosas yugales y de forma bilateral. En cuanto a su naturaleza, la ampolla es serosa, serofibrosa y hemorrágica o purulenta. Su contenido es fluido, viscoso o denso. Desde el punto de vista histológico, las ampollas pueden situarse dentro del epitelio y ser intraepiteliales (carácter más grave) o debajo del epitelio (subepiteliales) (14). Con todo esto, podrían distinguirse diferentes tipos de ampollas (Fig. 1): ampolla subepitelial, ampolla subcórnea y ampolla intraepitelial.

Por otro lado la vesícula o flictena puede ser multilocular y al punzar con un alfiler solo se vaciará parte de su contenido. Una vez roto el techo existe una pérdida de sustancia, formándose una lesión de tipo ulceroso $(2,6)$. En la práctica luego es muy difícil observar enteras las vesículas y ampollas para realizar un correcto diagnóstico de la lesión, ya que su vida como lesión clínica es corta y la humedad y traumatismos rompen rápidamente su techo. No obstante, su base es redondeada e irregular. Tampoco el tamaño nos hará diferenciar entre los dos tipos de lesiones, ya que los límites no siempre son precisos o definidos.

Como hemos dicho y de acuerdo con Grinspan la localización es importante para realizar un correcto diagnóstico (2). Las ampollas intraepiteliales ocurren por rotura de los desmosomas, creándose una cavidad a nivel del estrato espinoso. Este carácter anatomopatológico es importante para el diagnostico diferencial, ya que estas ampollas son de un carácter más grave. Representante de este grupo es el pénfigo (Tabla 1).

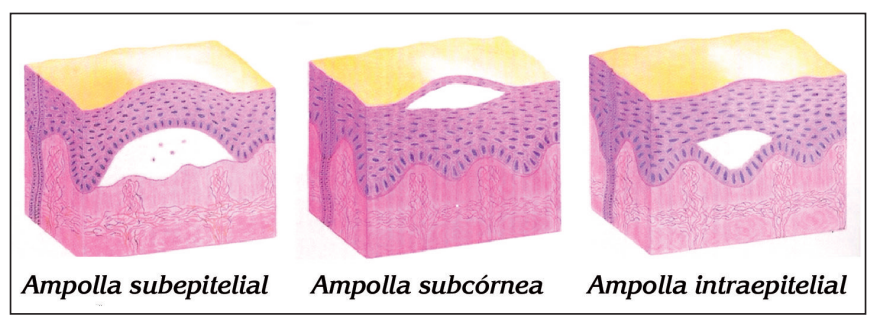

Fig. 1. Tipos de ampollas. 


\section{TABLA 1}

\section{FORMACIÓN DE AMPOLLAS (GRINSPAN, 1975)}

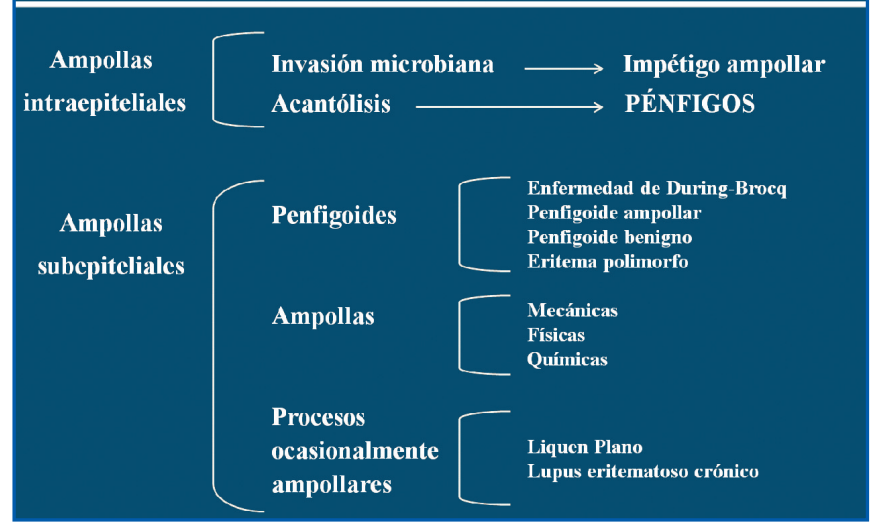

Con el nombre de pénfigo (del Griego "pemphix" o "akantha") se denomina a un grupo de enfermedades autoinmunes, que producen vesículas y ampollas a nivel cutáneo y de las membranas mucosas por la acción de autoanticuerpos contra proteínas específicas localizadas en las uniones de las células del epitelio $(4,5)$. Las ampollas son consecuencia de una pérdida de la adhesividad entre las células epidérmicas, proceso conocido con por acantólisis, y que se cree es causado por un proceso autoinmune $(6,7)$. Esta enfermedad es poco frecuente, de curso agresivo e evolución crónica, a brotes impredecibles de actividad $(8,66)$.

El pénfigo es una enfermedad grave autoinmune con manifestaciones cutáneo-mucosas cuya lesión elemental es una ampolla intraepitelial originada por la acantólisis $(26,67)$. La definición clásica determina que el pénfigo es una entidad de carácter grave, curso agresivo y evolución crónica, organoespecífica, en la que se producen vesículas y ampollas en piel y mucosas por la acción de autoanticuerpos contra proteínas específicas localizadas en las uniones de las células del epitelio y que requiere tratamiento continuo y sistemático para evitar su evolución letal (14).

Existen múltiples variantes clínicas del pénfigo. Las dos formas fundamentales son el pénfigo vulgar (PV) y el pénfigo foliáceo (PF). Estos dos grupos se diferencian clínica e histológicamente y por sus autoanticuerpos. El primero es la forma más común, con una participación importante en las mucosas.
El segundo se expresa en la piel (9). Otras variantes son el pénfigo vegetante y el pénfigo eritematoso. Además, han sido descritas nuevas entidades mucho más raras como son el pénfigo paraneoplásico (PPN), el pénfigo IgA y el pénfigo herpetiforme (10). Las manifestaciones orales son muy comunes en el pénfigo vulgar y en el pénfigo paraneoplásico, siendo muy infrecuentes en los otros tipos de pénfigo (11).

El diagnóstico de este tipo de lesiones se basa en una combinación de resultados clínicos e histopatológicos y se logra a través de técnicas de inmunofluorescencia directa (IFD), fundamentalmente realizadas en biopsias perilesionales de tejido afectado (12).

Dada la gravedad y cronicidad que pueden llegar a alcanzar estas enfermedades requieren tratamiento continuo y sistemático, a fin de evitar su evolución letal. Estos últimos años, el arsenal terapéutico ha sido extendido con la introducción de nuevas drogas y medicamentos, algunos de los cuales han cambiado el pronóstico de estas enfermedades (13).

En el presente artículo se pretende realizar una revisión y puesta al día de esta enfermedad, centrándonos en sus dos variantes que se expresan fundamentalmente con manifestaciones orales: el pénfigo vulgar y el pénfigo paraneoplásico. Se revisan los criterios etiopatológicos que nos llevan a comprender las manifestaciones clínicas, se analizan las formas de llegar al diagnóstico y los tratamientos que se están utilizando con mayor frecuencia en estos pacientes.

\section{EPIDEMIOLOGÍA}

El pénfigo es una enfermedad poco frecuente (14). La prevalencia descrita es de 0,5 a 3,2 casos por 100.000 habitantes (15) y la incidencia anual es de 0,1 a 0,5 casos por cada 100.000 habitantes $(16,17,67)$.

El pénfigo vulgar (PV) es la forma más común y grave de pénfigo y la que afecta a la mucosa oral con más frecuencia. En el $75 \%$ de los casos, las lesiones orales preceden a las cutáneas $(21,66)$. Se caracteriza por la presencia de ampollas intraepiteliales suprabasales, generadas por la presencia de acantóli- 
sis (14). Sigue un curso crónico, que puede producir afectación sistémica si no se trata de forma adecuada. El hecho de que en ocasiones sean las ampollas orales la primera manifestación de este desorden implica que los odontólogos deban conocer suficientemente las claves clínicas con el fin de realizar un diagnóstico y tratamiento precoces (67).

Afecta generalmente a personas que se encuentran en la cuarta o quinta décadas de vida. No presenta predilección racial o de género, pareciendo afectar a ambos sexos por igual. No obstante, algunos autores encuentran mayor predilección por el sexo femenino $(14,18,19)$.

Su incidencia máxima de ocurrencia se sitúa a partir de la cuarta y hasta la sexta décadas de la vida, si bien en ocasiones se observen casos en edades juveniles $(14,20)$.

Aunque la etiología y la patogénesis del PV no estén totalmente claras, parece haber un fondo o predisposición genéticos bastante fuertes. Esta enfermedad parece tener preferencia por la raza, ya que es más frecuente en determinados grupos étnicos. Grupos étnicos como los judíos de Ashkenazi (Europa Central y Oriental) y poblaciones de origen Mediterráneo e Indio son particularmente susceptibles (17, 22). Además, estos grupos étnicos con PV tienen una mayor expresión de HLA-DRW4 (complejo HLA de clase II) que la población general (25).

\section{ETIOPATOGENIA DEL PÉNFIGO}

El pénfigo es una enfermedad autoinmune que se caracteriza por tener anticuerpos IgG circulantes contra la desmogleína 3 (Dsg3) en la sustancia intercelular de los epitelios $(27,28)$. Alrededor de la mitad de los pacientes también tienen anticuerpos contra la Dsg1. Existe una cierta susceptibilidad, mayor con la presencia de factores de genes del HLA clase I (HLA-A10, HLA-A26) y sobre todo la asociación con moléculas de HLA clase II (DR4, DR14) (29).

Las ampollas se generan como consecuencia de la acción de autoanticuerpos circulantes contra proteínas presentes en los desmosomas que conforman la unión intercelular, provocando una pérdida de cohe- sión entre las células epidérmicas o los queratinocitos que se conoce por acantólisis y generando una ampolla. La ampolla puede ser intraepitelial, dando origen a lesiones en la mucosa; o intraepidérmica, dando origen a lesiones en la piel $(14,29)$.

El mecanismo de iniciación del PV no está claro, pero es probable que los factores genéticos puedan jugar un papel en la susceptibilidad a padecer la enfermedad. Lo confirman los casos de pénfigo descritos en familias, la mayor frecuencia de la enfermedad en determinados grupos étnicos como judíos y japoneses, o la asociación a genes del HLA (29).

La detección de estos auto-Ac en pacientes sanos, o la baja tasa de concordancia entre gemelos monocigóticos nos hace sospechar que los factores genéticos no son exclusivos en la etiología de la enfermedad (29). Se ha demostrado el papel etiológico de otros factores como son los ambientales, fármacos, virus, alimentos (cebolla, puerro, ajo) (39), quemaduras y exposición a rayos UV (29). Se ha demostrado que el pénfigo puede ser inducido por fármacos (40). Cualquier fármaco que posea un grupo activo tilo en su molécula se considera que puede inducir la aparición de este proceso. Algunos agentes conocidos son las sulfonamidas, las penicilinas y los anticonvulsivantes (40) También se han descrito casos de pénfigo asociado a otras enfermedades autoinmunes, como por ejemplo la artritis reumatoide, la miastenia grave, el lupus eritematoso y la anemia perniciosa (30).

Como se ha mencionado antes, el pénfigo es una enfermedad autoinmune organoespecífica donde se producen autoanticuerpos contra antígenos localizados en los desmosomas de los queratinocitos, rompiendo la adhesión intercelular, llevando a la acantólisis y a la formación de ampollas intraepiteliales. Estos autoanticuerpos son fundamentalmente IgG, sobre todo IgG1, IgG4, IgG2 y IgG3, y con mucha menor frecuencia IgM. Aunque la patogenicidad del pénfigo está caracterizada por la producción de estos autoanticuerpos es probable que la respuesta de la inmunidad celular también juegue un importante papel. La asociación con moléculas de HLA clase II (DR4, DR14) va a facilitar la presentación de péptidos derivados de la desmogleína a clones específicos de linfocitos T CD4+, que segregan citoquinas Th2 (IL4, 
IL6, IL10), mediando estas en la producción de anticuerpos patógenos por las células B (29).

Se ha sospechado del papel patógeno de estos autoanticuerpos (auto-Ac) por la correlación existente entre la actividad de la enfermedad y los títulos de estos en la sangre (siendo la recaída más frecuente en pacientes con títulos persistentes y elevados) y por los casos de pénfigo descritos en neonatos de madres con enfermedad activa durante el parto (33). Los casos en los que hay regresión de la enfermedad con la eliminación espontánea de los anticuerpos maternos son casos muy útiles para demostrar el papel patógeno de los auto-Ac en modelos de experimentación tanto animales como in vitro, mediante proteínas purificadas $(29,31)$.

Estos auto-Ac van dirigidos contra unos antígenos epiteliales que se han identificado en el PV fundamentalmente con las moléculas de adhesión desmogleínas 3 (glucoproteína de 130 KD responsable mayor de la adhesión de las células en la mucosa oral) que pertenece a la familia de las cadherinas y están localizadas en las uniones del estrato espinoso, produciéndose la acantólisis en capas profundas del epitelio. Por otro lado, en el PF estos antígenos se han identificado con las desmogleinas 1 (160 KD), localizadas en la capa granular del epitelio, produciendo así acantólisis en capas más superficiales del epitelio $(28,30)$.

La causa por la que la tolerancia inmunológica se rompe contra estas proteínas permanece desconocida. Recientes hallazgos aportan argumentos a favor de una inhibición específica de la función adhesiva de la desmogleína 1 y 3 por los correspondientes anticuerpos (Ac) en los diferentes tipos de pénfigo, explicando la distinta localización de las lesiones observadas en el pénfigo foliáceo, pénfigo vulgar mucoso, y pénfigo vulgar con participación mucocutánea.

\section{CARACTERÍSTICAS CLÍNICAS DE PÉNFIGO}

El $60 \%$ de los pénfigos tienen inicio en la cavidad oral (35). La mucosa oral está afectada la mayoría de las veces (90\%) y sólo el 10-15\% tienen afectación exclusiva cutánea. Es infrecuente en la laringe, faringe, esófago, uretra, vulva, pene, ano, cérvix, recto y conjuntiva, pero es muy grave cuando se da en estas localizaciones (23).

Existen varias formas de pénfigos, en función de la evolución y del tipo de anticuerpo detectado (Tabla 2). Como hemos ya referido anteriormente, el pénfigo vulgar (PV) es la forma clínica más común.

El pénfigo vulgar (PV) se expresa con lesiones orales en un $88 \%$ de los pacientes (23). Generalmente comienza con lesiones muy inespecíficas, pasando meses desde el inicio hasta su diagnóstico. El diagnóstico suele ser tardío. La mayoría de los pacientes presentan síntomas dos a seis meses antes de llegar al diagnóstico definitivo $(23,66)$.

La lesión elemental son las ampollas, que suelen ser múltiples, mal definidas, de distinto tamaño, de techo fino que se rompen fácilmente produciendo erosiones, superficiales, irregulares y muy dolorosas. Otros signos concomitantes incluyen la formación de nuevas ampollas junto con otras ya evolucionadas y úlceras, expresando un carácter progresivo (23, 67). Por lo tanto, podemos observar ampollas integras, ampollas en las que el techo de las mismas se está desprendiendo y aparece como una auténtica membrana de tejido organizado que se puede separar con una sonda; o pseudomembranas, que cubren erosiones, formadas fundamentalmente por un exudado inflamatorio que no constituye un tejido organizado que pueda separarse con un explorador (3).

Cualquier localización de la mucosa oral puede estar afectada, pero con mayor frecuencia lo están las áreas de roce como es la mucosa yugal cerca del plano oclusal (Figs. 2, 3A y 3B), los labios, la encía

\section{TABLA 2.- CLASIFICACIÓN DE LOS PÉNFIGOS}

- Pénfigo vulgar.

- Pénfigo vegetante tipo Newman.

- Pénfigo vegetante tipo Hallopeau.

- Pénfigo foliáceo.

- Pénfigo eritematoso (Lever).

- Pénfigo paraneoplásico. 


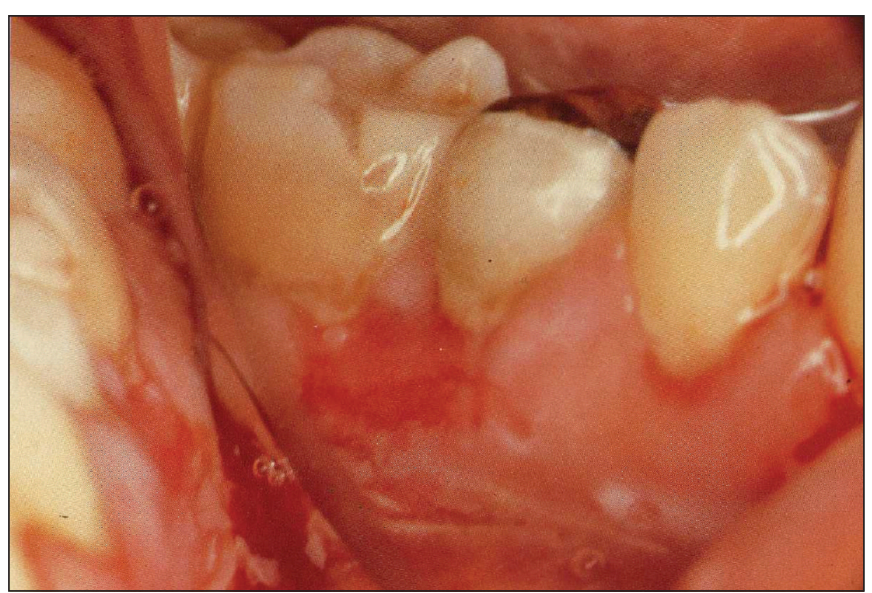

Fig. 2. Pénfigo vulgar en mucosa gingival. Presentación oral típica con eritema, erosiones y úlceras persistentes. El epitelio alrededor es friable y se desintegra cuando presionado gentilmente.
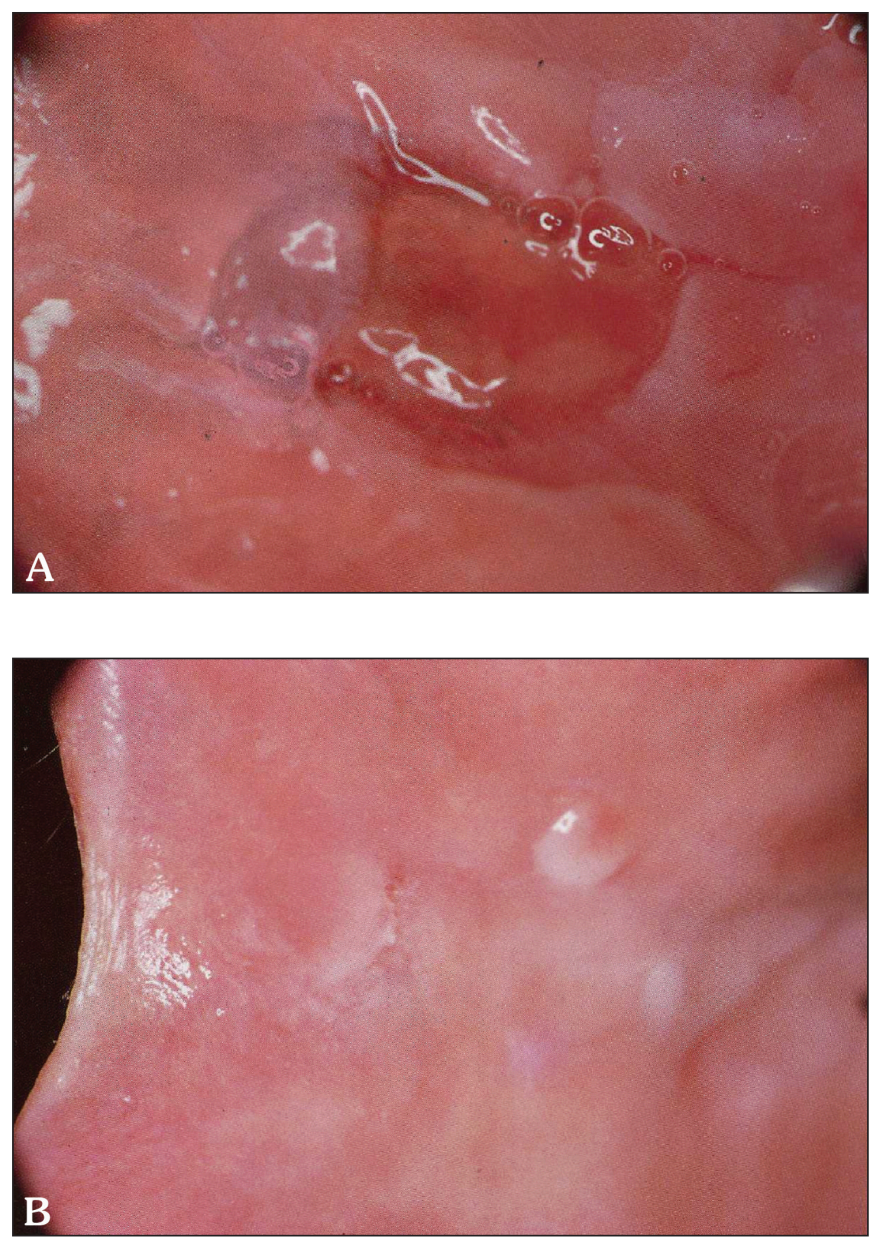

Fig. 3. A: Pénfigo vulgar en la Mucosa yugal. B: El mismo caso después de la inyección perilesional de corticoides. alveolar edéntula y el paladar blando (Figs. 4 y 5) (23). Las erosiones tienen tendencia a extenderse por toda la cavidad oral a partir de sus bordes, con un importante componente eritematoso (5). En los labios, las erosiones evolucionan a costras serohemáticas. Las lesiones curan sin dejar cicatrices. En estos pacientes el signo de Nikolsky es positivo, ya que cualquier presión o traumatismo en la periferia de la lesión es capaz de incrementar rápidamente el tamaño inicial $(5,36)$. En algunas ocasiones la participación de la encía se manifiesta como una gingivitis descamativa: encías eritematosas, brillantes, finas, con vesículas en la superficie que al rozarlas levemente se desprende el epitelio quedando expuesto el conectivo adyacente hemorrágico, expresando su carácter descamativo y erosivo (Fig. 6) (11).

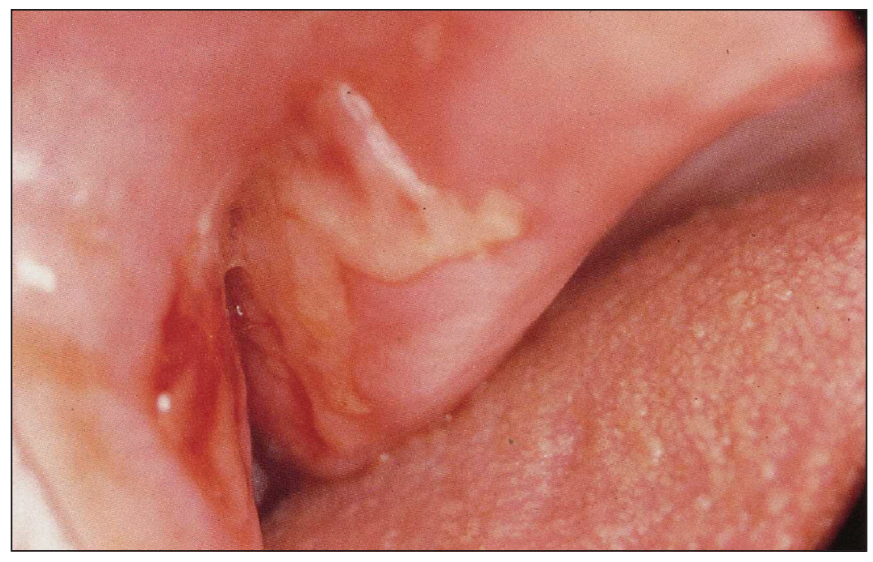

Fig. 4. Pénfigo vulgar en región gingival edéntula.

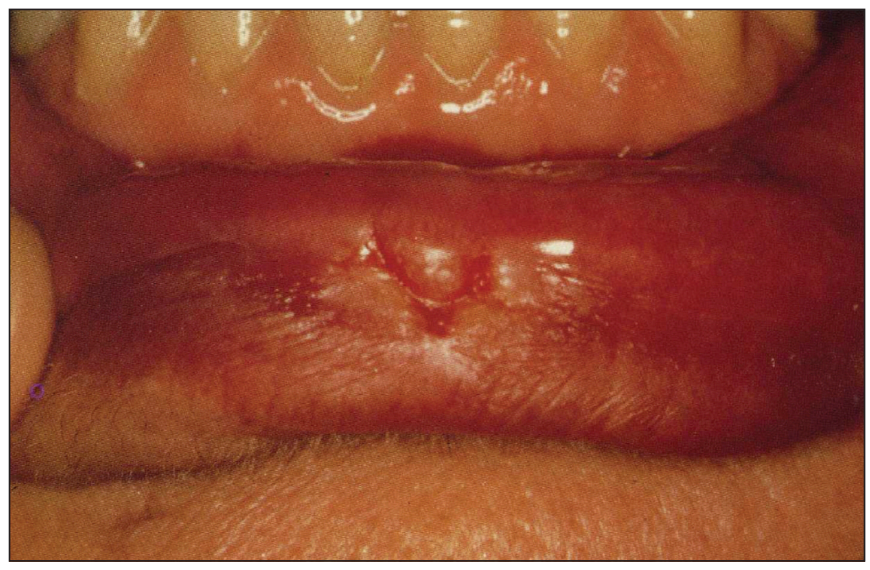

Fig. 5. Pénfigo vulgar en región anterior labial. 


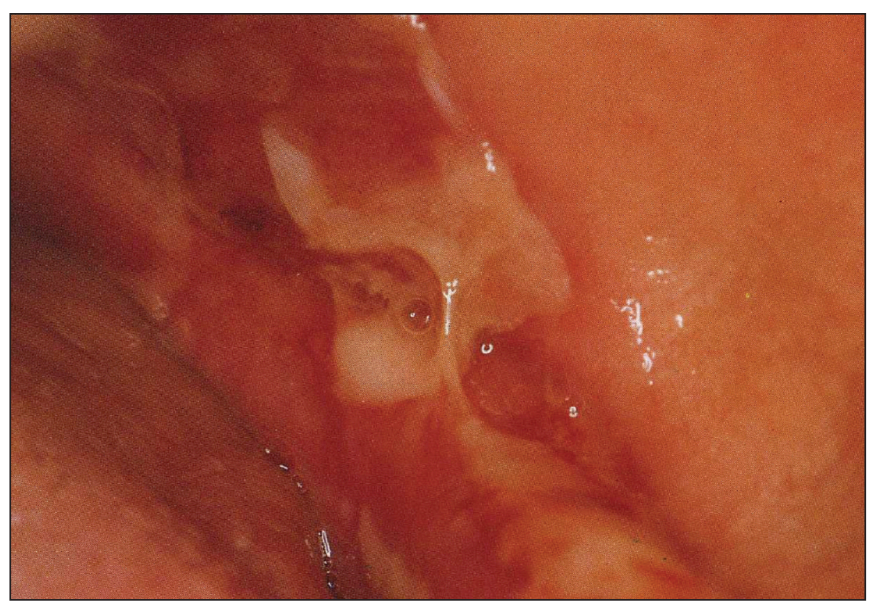

Fig. 6. En algunas ocasiones la participación de la encía se manifiesta como una gingivitis descamativa crónica.

Cuando el pénfigo tiene manifestaciones en la piel, estas suelen aparecer meses o años después de las lesiones orales. Se manifiestan como ampollas sobre una piel normal o ligeramente eritematosa, localizadas en cualquier parte del cuerpo pero con mayor frecuencia en cuero cabelludo, cara y parte superior del tronco (Fig. 7) $(36,67)$.

En un estudio publicado por Scully y cols en 1999, se determina que una cuarta parte de los pacientes con lesiones en la cavidad oral desarrollan, en el transcurso del tiempo, lesiones en la piel, que llegan a manifestarse meses o años tras las lesiones intraorales (23).

También pueden afectarse otras mucosas como la faringe, la nariz, la laringe, el esófago, la uretra, la vulva, el cérvix, el pene, el ano, el recto y la conjuntiva, aunque con mucha menor frecuencia y mayor gravedad $(4,11,21,37)$.

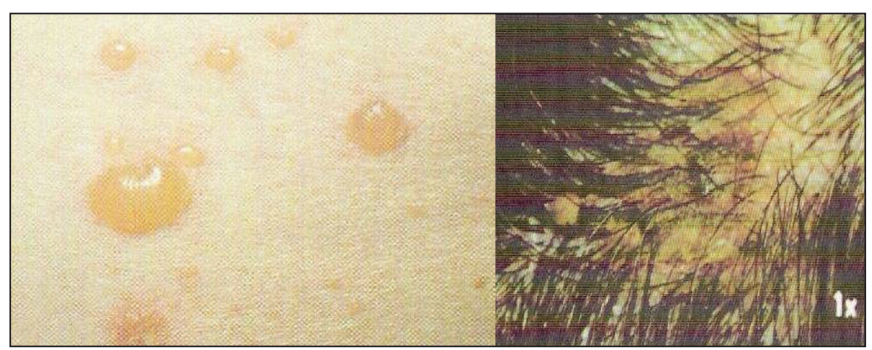

Fig. 7. Pénfigo vulgar. Lesiones ampollosas en la piel y cuero cabelludo.
El pénfigo paraneoplásico (PPN) es mucho menos frecuente que el PV, pero presenta una gran frecuencia e intensidad con que se manifiesta en la cavidad oral (58).

Fue descrito inicialmente por Anhalt y cols. en 1990. Se define como una enfermedad autoinmune causada por una alteración linfoproliferativa subyacente, con unos criterios diagnósticos clínicos, histológicos, e inmunológicos que lo definen y diferencian del PV (59).

Clínicamente los PPN se caracterizan por una severa afectación oral que consiste en una estomatitis y lesiones polimorfas cutáneas que afectan preferentemente al tronco, extremidades, palmas y plantas en un paciente con una neoplasia oculta o confirmada.

Aproximadamente dos tercios de los casos de PPN ocurren en pacientes con historia y diagnóstico de neoplasia. En el tercio restante la lesión neoplásica se detecta después de la enfermedad mucocutánea (10).

El PPN se asocia con mayor frecuencia a neoplasias hematológicas ( $84 \%$ de los casos) sobre todo linfomas no-Hodgkin (42\%) (61), leucemias linfocitarias crónicas (29\%), enfermedad de Castleman (10\%) y otras. También se han descrito casos de PPN asociado a neoplasias no hematológicas ( $16 \%$ de los casos) (64).

Existe una participación de la mucosa oral importante en forma de mucositis dolorosa y persistente en todos los casos descritos y es el primer signo de la enfermedad en el $45 \%$ de los pacientes. En algunos pacientes solo existe afectación de la orofaringe sin llegar manifestarse en la piel (59). La estomatitis se manifiesta con erosiones y ulceraciones difusas severas, persistentes, muy dolorosas, y extremadamente resistentes al tratamiento y con un importante componente necrótico. Pueden afectar cualquier localización de la cavidad oral, sobre todo los bordes laterales de la lengua y característicamente existe también participación del borde rojo de los labios. También se observa una participación importante de la faringe aunque pueden aparecer lesiones en todas las mucosas del organismo presentando la mayoría de los pacientes una severa conjuntivitis pseudomembranosa (59). 
Las lesiones cutáneas son variables y polimorfas observándose ampollas, erosiones y lesiones en diana, recordando en ocasiones un cuadro de eritema multiforme o necrosis epidérmica tóxica por su gravedad. Es característica la localización en palmas y plantas de los pies.

El PPN es el único tipo clínico de pénfigo que se manifiesta en tejidos que no están tapizados por epitelio escamoso estratificado desarrollando en aproximadamente un $30-40 \%$ de los casos lesiones pulmonares que pueden llevar a un fatal desenlace $(59,60)$.

\section{DIAGNÓSTICO DE PÉNFIGO}

El diagnóstico clínico de presunción ha de ser confirmado cuanto antes por estudios complementarios, que varían ampliamente en su especificidad y en su complejidad, entre los que se destacan: la citología exfoliativa, la histopatología, la inmunofluorescencia directa (IFD), la inmunofluorescencia indirecta (IFI) y los estudios de inmunoprecipitación $(14,66)$.

En el pénfigo vulgar, la citología exfoliativa (biopsia) tomada en fase de ampolla o vesícula muestra las células acantolíticas de Tzanck que son células epiteliales libres, redondeadas, de bordes desflecados y núcleos hipercromáticos, con citoplasma homogéneo eosinófilo en una tinción de Papanicolau (Fig. 8). No obstante, estas células no son patognomóni-

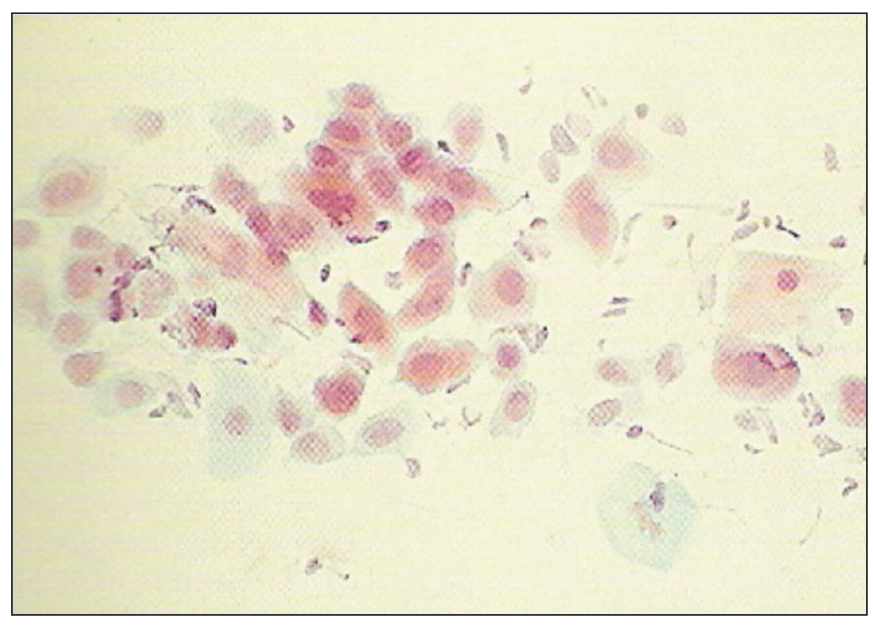

Fig. 8. Citodiagnóstico de Tzank. Células acantolíticas de Tzanck. cas del PV y pueden observarse también en otras enfermedades ampollares (40).

Además de las características clínicas y citológicas, lo que distingue a un pénfigo es la presencia de rasgos histopatológicos diferenciales. En el estudio histológico del PV observamos inicialmente un edema intercelular en los estratos suprabasales del espinoso con la formación de hendiduras y acantólisis, que llevan a la formación de ampollas (Figs. 9 y 10) (34, 67). Es útil para observar la presencia de células acan-

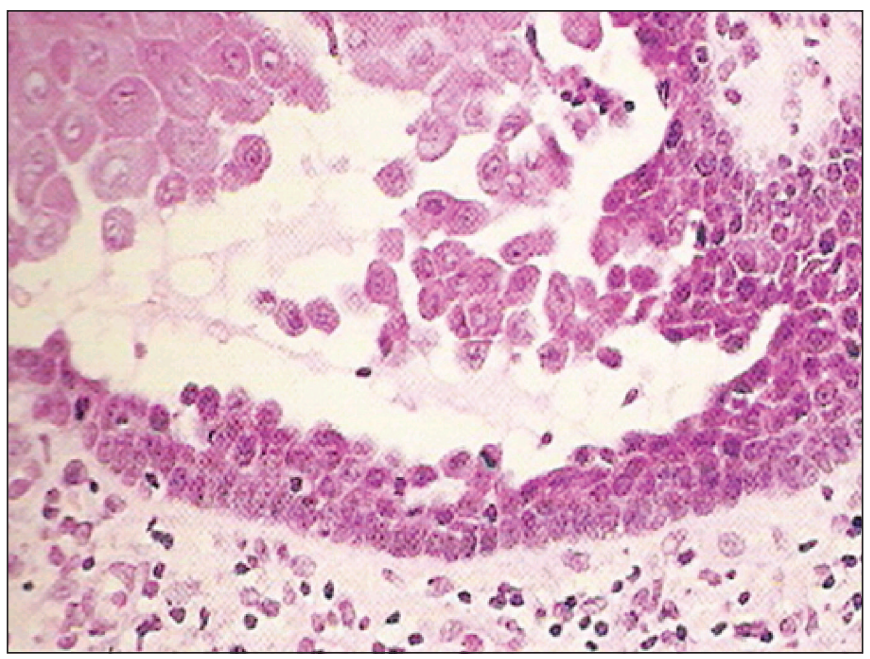

Fig. 9. Ampolla intraepitelial en una lesión de pénfigo.

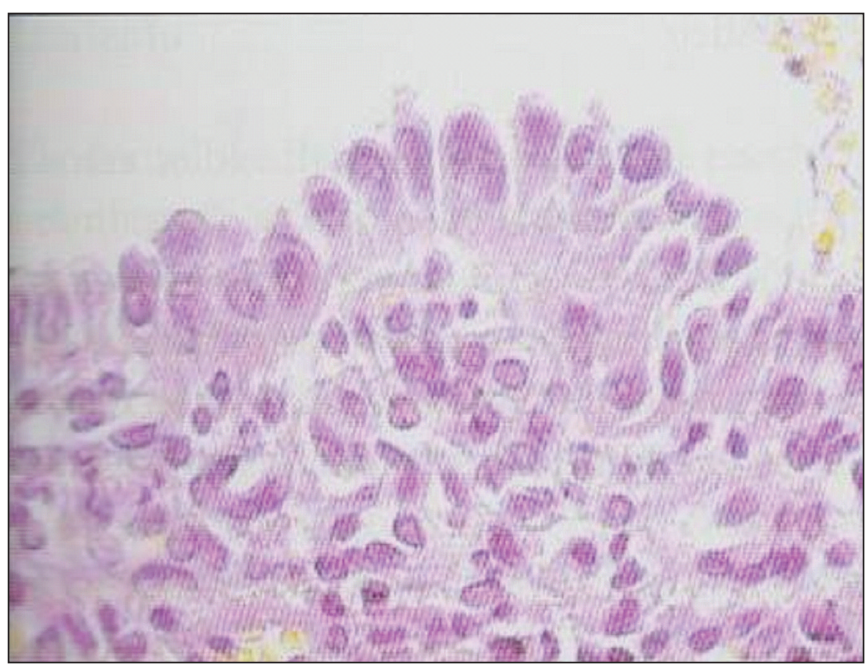

Fig. 10. Suelo de una ampolla intraepitelial en una lesión de pénfigo, observándose la denominada imagen en "cementerio inglés". 
tolíticas flotando en el interior del estrato espinoso del epitelio, pero la inmunofluorescencia directa (IFD) es necesaria para confirmar el diagnóstico.

Los estudios de inmunofluorescencia directa (IFD) muestran el marcaje en la superficie de las células epiteliales, indicando depósito de inmunoglobulinas en la membrana de los queratinocitos (espacio intercelular). Se observa en el $100 \%$ de los casos depósito de IgG en los espacios intercelulares del epitelio y mucho menos frecuente IgM. También puede detectarse depósito de complemento con la misma distribución (especialmente la fracción C3) (29).

Los resultados encontrados a través de la inmunofluorescencia indirecta (IFI) son parecidos a la directa. Se detecta anticuerpos circulantes antisuperficie de las células epiteliales (antisustancia intercelular) fundamentalmente IgG4 e IgG1, y menos frecuentemente la IgG3, Se ha comprobado que existe una cierta correlación entre el título de anticuerpos y el estadio clínico de la enfermedad y son pues útiles para evaluar su evolución y el tratamiento. La recuperación se correlaciona con la disminución de los títulos de IgG4 permaneciendo la IgG1 positiva, demostrando el importante papel patógeno de la IgG4 (29). En ocasiones cuando la enfermedad está vinculada a la contraceptivos orales es dificil encontrar anticuerpos circulantes, lo que también sucede cuando el paciente está en fase inactiva por tratamiento con corticoides.

En la actualidad los estudios de inmunoprecipitación son considerados uno de los más definitivos e identifican los antígenos diana reconocidos por los autoanticuerpos del paciente, reconociéndose en el PV la Dsg3; en el PF, la Dsg1; y en el PPN plakinas. Esta es una asociación que facilita la descripción de la enfermedad. No obstante, algunos antígenos son reconocidos en diferentes tipos de pénfigo. Por ejemplo la Dsg1 y la Dsg3 pueden ser detectadas en el suero de los pacientes con PV. Además, se ha demostrado que este solapamiento de antígenos está relacionado con las manifestaciones clínicas del PV. El suero de pacientes de PV con manifestaciones clínicas exclusivas en mucosas solo contienen IgG anti Dsg3, mientras que el suero de pacientes con manifestaciones mucocutáneas reaccionan contra la Dsg1 y la Dsg3 (4, 59).
En suma, el diagnóstico de PV se realiza por la sospecha clínica de las lesiones, pero ha de ser en todos los casos confirmado por la presencia de acantólisis en la biopsia y por la determinación de anticuerpos, bien en la sangre o en los tejidos.

El pénfigo paraneoplásico se evidencia histológicamente como un proceso cutáneo liquenoide, a menudo combinado con un despegamiento intraepitelial.

La IFD detecta IgG y complemento localizados en el espacio intercelular del epitelio junto con depósito lineal o granular del complemento en la zona de la membrana basal. Se detectan también autoanticuerpos séricos que se unen no solo a la superficie de las células del epitelio escamoso de piel y mucosas como en el pénfigo, sino también al epitelio simple, columnar y transicional, detectados por IFI y siguiendo el patrón típico del pénfigo (positiva para los Ac antisustancia intercelular) (60).

Los estudios de inmunoprecipitación reconocen la presencia de antígenos específicos de los desmosomas y hemidesmosomas: desmoplakina I (250KD), BPAg (230KD), envoplakin y desmoplakin II (210KD), periplakin (190KD) y un Ag indeterminado de 170 KD y la desmogleína 1 y $3(58,59)$.

En una revisión del PPN realiza por Camisa y Helm en 1993 se dividen los criterios diagnósticos del PPN en criterios mayores y criterios menores (Tabla 3) (62). Para el diagnóstico de PPN se requieren tres criterios mayores o dos menores y dos mayores $(58,63)$.

Las características histopatológicas del PPN son variables. Reflejan el polimorfismo de sus manifestaciones clínicas, con acantólisis intraepitelial suprabasilar, necrosis de queratinocitos y degeneración vacuolar entre las más frecuentes.

En la IFD se detectan depósitos intercelulares de IgG y $\mathrm{C}$ y depósito del complemento en la zona de la membrana basal, lo que permite el diagnóstico diferencial con lesiones de PV. Es más frecuente observar falsos negativos en los resultados de inmunofluorescencia en el PPN que en el PV, de aquí la necesidad de repetir las biopsias. 


\section{TABLA 3.- CRITERIOS DIAGNÓSTICOS DEL PPN}

Criterios mayores:

- Severa afectación oral y cutánea polimorfa.

- Neoplasia interna.

- Anticuerpos determinados por inmunoprecipitación.

Criterios menores:

- Patrón histológico de acantolisis.

- IFD mostrando depósito de IgG marcando los espacios intercelulares y la zona de la membrana basal.

- Anticuerpos séricos detectados por IFI.

\section{TRATAMIENTO DEL PÉNFIGO}

En el tratamiento de este tipo de patologías, resulta útil que el Odontólogo esté en estrecha conexión con el Dermatólogo y el Internista, para un correcto seguimiento de los casos.

El tratamiento del pénfigo en general y del PV en particular va dirigido a detener la producción de los anticuerpos agresores, que producen daño tisular (41). En el momento actual no existe ninguna terapia que suprima específicamente los anticuerpos antidesmogleína, por lo que el tratamiento sigue basado en la inmunosupresión no específica.

Los fármacos de elección de los que disponemos para el tratamiento del pénfigo son los corticoides (67). La introducción de los corticoides disminuyo drásticamente las tasas de mortalidad de estos pacientes. Sin embargo, las altas dosis y el tiempo prolongado de la utilización de los corticoides llevan a la aparición de graves efectos secundarios. Los efectos adversos más frecuentes de los corticoides son la osteoporosis, el empeoramiento o desencadenamiento de diabetes y la hipertensión (44). Normalmente, la supresión de estos se consigue administrando dosis moderadas por vía oral $(42,67)$.

El tratamiento inicial del pénfigo vulgar requiere el uso de corticoides sistémicos. Se puede iniciar el tratamiento con dosis de prednisona de $1 \mathrm{mg} / \mathrm{kg} /$ día $\mathrm{y}$, una vez controlado el brote y a medida que se observan resultados positivos, ir reduciendo progresivamente la dosis, hasta pasar a régimen de administración a días alternos. Esto permite minimizar los efectos secundarios del fármaco, hasta llegar a alcanzar dosis mínimas de mantenimiento $(46,47)$. Los fármacos que podemos utilizar son la fluocinolona al $0,05 \%$ o propionato de clobetasol al 0,05\%. El tratamiento local con corticoides tópicos tiene un potente efecto antiinflamatorio. La vía tópica se aplica como coadyuvante a la administración por vía oral o como terapia de mantenimiento. En casos de lesiones localizadas a nivel gingival, pueden utilizarse las férulas oclusales para administrar los corticoides (48).

Si la monoterapia con corticoides no controla las lesiones, no impide las recaídas o produce efectos secundarios importantes está indicada la terapia combinada. La utilización de terapias adyuvantes con agentes inmunosupresores (como la azatioprina, ciclofosfamida, metrotexato, ciclosporina) y el empleo de nuevos agentes (como el mofeti micofenolato l o el tacrolimus) parecen constituir alternativas inmunosupresoras en monoterapia o terapia combinada con bajas dosis de corticoides (Tabla 4). La terapia combinada suele iniciarse con la suma de inhibidores de la síntesis de la purina $(43-45,67)$ o el tracolinus.

En pacientes con gran agresividad clínica o rápido deterioro del estado general se ha utilizado la Plasmaféresis como terapia adyuvante junto con la administración de corticoides e inmunosupresores. La Plasmaféresis es la terapia más directa para reducir la cantidad de anticuerpos patógenos en el suero. Esta reducción produce un efecto rebote en la síntesis de Ac por los linfocitos B debido a la eliminación del mecanismo de feedback. Sin embargo esto se puede frenar con la administración de ciclofosfamida ya que produce una inhibición de la proliferación de linfocitos (53).

También se ha utilizado la administración de inmunoglobulinas intravenosas. Estas pueden provocar una rápida reducción de los autoanticuerpos de la circulación pero provocan un importante aumento en el catabolismo proteico y una mejoría a corto plazo no alargando los periodos de remisión (54). 
TABLA 4.- TERAPIAS ADYUVANTES ALTERNATIVAS INMUNOSUPRESORAS EN MONOTERAPIA O TERAPIA COMBINADA CON BAJAS DOSIS DE CORTICOIDES UTILIZADAS EN EL PV

\begin{tabular}{|c|c|c|c|}
\hline Fármaco & Dosis recomendada & Propiedades & Efectos secundários \\
\hline $\begin{array}{l}\text { Mofetil } \\
\text { micofenolato }\end{array}$ & 2g/día & $\begin{array}{l}\text { - Mayor perfil de seguridad. } \\
\text { - Desarrolla menos efectos secunda- } \\
\text { rios: inicio de acción lento requi- } \\
\text { riendo a menudo de dos a tres } \\
\text { meses para el control de los anti- } \\
\text { cuerpos. } \\
\text { - Terapia cara, no siempre efectiva } \\
(43,44) \text {. } \\
\text { - Necesita monitorización sanguínea } \\
\text { con un recuento hemático periódi- } \\
\text { co }(50) \text {. }\end{array}$ & $\begin{array}{l}\text { - Fatiga. } \\
\text { - Intolerancia ocasional } \\
\text { debido al dolor óseo. } \\
\text { - Linfopenia. } \\
\text { - Alteraciones gastroin- } \\
\text { testinales. } \\
\text { - Aumento de la inciden- } \\
\text { cia de infecciones (49). }\end{array}$ \\
\hline Azatioprina & $\begin{array}{l}\text { * Pacientes con niveles } \\
\text { normales de TPMT: } \\
3 \mathrm{mg} / \mathrm{kg} / \text { día. } \\
\text { Actividad antiinflamato- } \\
\text { ria con dosis de } 2 \mathrm{mg} / \mathrm{k} / \\
\text { día (pero no es eficaz } \\
\text { para la inhibición de la } \\
\text { producción de auto-Ac) } \\
(44,45,49) \text {. }\end{array}$ & $\begin{array}{l}\text { - Más segura para alcanzar el control } \\
\text { de la enfermedad. } \\
\text { - Coste más bajo. } \\
\text { - Mayor incidencia de efectos secun- } \\
\text { darios, que disminuye determinan- } \\
\text { do la actividad del enzima tiopurina } \\
\text { metil transferasa (TPMT, enzima } \\
\text { que metaboliza la azatioprina). Se- } \\
\text { gún la actividad, nos indica la do- } \\
\text { sis ajustada para cada paciente*. }\end{array}$ & $\begin{array}{l}\text { - Leucopenia. } \\
\text { - Pancitopenia. } \\
\text { - Hepatotoxicidad. } \\
\text { - Nauseas. } \\
\text { - Fiebre. }\end{array}$ \\
\hline Ciclofosfamida & $\begin{array}{l}2 \text {-3 mg/kg/día junto con } \\
\text { una intensa hidratación } \\
\text { Terapia pulse: } \\
100 \text { mg de dexametaso- } \\
\text { na en perfusión IV lenta } \\
\text { durante tres días conse- } \\
\text { cutivos, junto } 500 \text { mg de } \\
\text { ciclofosfamida aplicada } \\
\text { un solo día, esto se repi- } \\
\text { te una vez al mes (52). }\end{array}$ & $\begin{array}{l}\text { - Agente alquilante utilizado de ma- } \\
\text { nera muy efectiva en el tratamiento } \\
\text { del pénfigo. } \\
\text { - Estos pacientes necesitan un segui- } \\
\text { miento con recuento hemático y } \\
\text { analítica urinaria semanalmente al } \\
\text { principio y cada dos semanas pos- } \\
\text { teriormente. } \\
\text { - Administración en terapia pulse (in- } \\
\text { fusión intravenosa discontinua de } \\
\text { dosis muy altas de fármaco en un } \\
\text { periodo corto de tiempo) disminu- } \\
\text { ye su potencial tóxico al disminuir } \\
\text { la acumulación del fármaco y así } \\
\text { los efectos secundarios (tratamien- } \\
\text { to menos tóxico pero también me- } \\
\text { nos efectivo) (45). }\end{array}$ & $\begin{array}{l}\text { - Gran toxicidad tem- } \\
\text { prana (mielosupresión } \\
\text { y cistitis hemorrágica) } \\
\text { y tardía (aumenta el } \\
\text { riesgo de padecer lin- } \\
\text { fomas, leucemias y } \\
\text { cáncer de vejiga). } \\
\text { - Asociada con un ma- } \\
\text { yor riesgo de esterili- } \\
\text { dad. }\end{array}$ \\
\hline
\end{tabular}


La ausencia de estudios controlados a largo plazo para valorar la eficacia de los tratamientos ha llevado a la utilización de una amplia variedad de fármacos en el pénfigo. Entre los fármacos utilizados están el metotrexate, la dapsona, agentes antimaláricos, y terapia con oro también designada crisoterapia (5557). Estos agentes no reducen la síntesis de Ac, por lo que su eficacia es cuestionable (44).

El tratamiento de los pacientes con PPN está condicionado en parte por la neoplasia asociada. Los pacientes con tumores resecables quirúrgicamente, como tumores benignos, tumor de Castleman o timomas, curan o mejoran ampliamente tras la resección del mismo. La curación completa se observa de 6 a 18 meses tras la escisión del tumor (65).

El PPN asociado a una neoplasia maligna generalmente no se resuelve a pesar del control o cura de la neoplasia. Esto nos indica que una vez que la enfermedad autoinmune se desencadena puede progresar de manera independiente.

No hay evidencia de una pauta de tratamiento efectivo en pacientes con diagnóstico de PPN, describiéndose que con corticoides orales a dosis de 0,5$1 \mathrm{mg} / \mathrm{kg}$ pueden mejorar las lesiones (60). Por esto en estos pacientes han utilizado una multitud de tratamientos fallando en la mayoría de ellos. Estos tratamientos han incluido inmunosupresión con ciclofosfamida, azatioprina, oro, dapsona, plasmaféresis, inmunoglobulinas intravenosas a altas dosis y terapia pulse con ciclofosfamida, tal y como hemos descrito anteriormente (60).

El fallecimiento de estos pacientes se ha relacionado con múltiples causas entre ellas sepsis, hemorragias gastrointestinales, fallo multiorgánico y fallo respiratorio (59).

\section{EVOLUCIÓN Y PRONÓSTICO}

Para la evolución de las lesiones de pénfigo son indicadores pronósticos la edad del paciente al inicio de la enfermedad, la extensión de las lesiones y las dosis necesarias de corticosteroides utilizadas en el tratamiento. No obstante, el curso de la enfermedad es variable e imprevisible (14).
Antes de la introducción de los corticoides la historia natural del pénfigo era hacia la extensión progresiva, presentando una tasa de mortalidad a los dos años del $50 \%$ y del $100 \%$ a los cinco años. Se estima que lo que sucedía era muerte en el $10 \%$ de los casos, $40 \%$ se curaban y no necesitaban tratamiento, $20 \%$ requerían dosis bajas de corticosteroides de mantenimiento y $30 \%$ tenían recurrencias continuas (38). El uso de los corticoides redujo la tasa de mortalidad a menos del 5\%. Sin embargo sigue estando asociado a una importante tasa de morbilidad por su utilización prolongada (44).

La mayoría de los pacientes necesitan tratamiento continuo con fármacos inmunosupresores. La eficacia del tratamiento se valora primariamente por la mejoría clínica y secundariamente por los parámetros de laboratorio (disminución de los Ac séricos). Actualmente no existen criterios estandarizados para valorar la mejoría del cuadro de pénfigo, pero los criterios clínicos que le determinan son la ausencia de nuevas lesiones, curación de las existentes y un signo de Nikolsky negativo (45).

El pénfigo puede evolucionar de manera variable hacia el control de la enfermedad sin requerir terapia de mantenimiento, hacia la necesidad de administrar una terapia de mantenimiento a dosis mínima (corticoides junto inmunosupresores), o incluso a la muerte del paciente (23).

Hoy en día la mayor causa de mortalidad del pénfigo sigue siendo las complicaciones del tratamiento sistémico con los corticoides y los inmunosupresores, por lo que se necesita una cuidadosa monitorización de estos fármacos, siendo las infecciones la principal causa de mortalidad $(23,24)$. Es importante resaltar el valor del diagnóstico y tratamiento precoz de estos pacientes, ya que mejora el pronóstico y curso de la enfermedad. También aquellos pacientes con una rápida respuesta al tratamiento tienen mejor pronóstico (21).

\section{BIBLIOGRAFÍA}

1. McCuin JB, Hanlon T, Mutasim DF. Autoimmune bullous diseases: diagnosis and management. Dermatol Nurs. 2006 Feb;18(1):20-5. 
2. Grinspan D. Semiología y propedéutica clínica. Buenos Aires: Mundi, 1975.

3. Bermejo-Fenoll A, López-Jornet P. Diagnóstico de las enfermedades vesiculares y ampollares de la mucosa bucal: desórdenes de la cohesión intraepitelial y de la unión epitelioconectiva. Med Oral 1996;1:24-43.

4. Weinberg MA, Insler MS, Campen RB. Mucocutaneous features of autoinmune blistering diseases. Oral Med Oral Pathol Oral Radiol Endod 1997;84:517-34.

5. Bagán JV. Enfermedades ampollares de la cavidad oral (I): pénfigos. En: Bagán JV, Ceballos A, Bermejo A, Aguirre JM, Peñarrocha M, eds. Medicina Oral. Barcelona: Masson, 1995:220-6.

6. Bianciotto C, Herreras Cantalapiedra JM, Alvarez MA, Méndez Díaz Mc. Conjunctival blistering associated with Pemphigus Vulgaris: Report of a case. Arch Soc Esp Oftalmol. 2005 Jun;80(6):365-8.

7. Femiano F. Seeking approval: present and future therapies for pemphigus vulgaris. Curr Opin Investig Drugs. 2008 May;9(5):497-504. Review.

8. Camacho-Alonso F, López-Jornet P, BermejoFenoll A. Pemphigus vulgaris. A presentation of 14 cases and review of the literature. Med Oral Patol Oral Cir Bucal. 2005 Aug-Oct;10(4):282-8.

9. Jiménez-Soriano Y, Díaz-Fernández JM. Enfermedades ampollares en la cavidad oral: pénfigo. RCOE 2004;9(4):439-447.

10. Robinson ND, Hashimoto T, Amagai M, Chan LS. The new phemphigus variants. J American Academy Dermatol 1999;40:649-71.

11. Scully, Paes de Almeida, Porter, Gilkes. Pemphigus vulgaris: the manifestations and long term managenent of 55 patients with oral lesions. British J Dermatol 1999;140:84-92.

12. Guillen S, Khachemoune A. Pemphigus vulgaris: a short review for the practitioner. Dermatol Nurs. 2007 Jun; 19(3):269-72.
13. Suárez-Fernández R, España-Alonso A, HerreroGonzález JE, Mascaró-Galy JM. Practical management of the most common autoimmune bullous diseases. Actas Dermosifiliogr. 2008 Jul; 99(6):441-55.

14. Antonio Bascones Martínez, Felipe Llanes Menéndez. Medicina Bucal. Madrid. Ediciones Avances Médico-Dentales, 1991.

15. Smith RJ, Manche EE, Mondino BJ. Ocular cicatricial pemphigoid and ocular manifestations of pemphigus vulgaris. Int Ophthalmol Clin 1997; 37:63-75.

16. Becker BA, Gaspari AA. Pemphigus vulgaris and vegetants. Dermatol Clin. 1993;11:429-52.

17. Joly P. Incidence of bullous pemphigoid and pemphigus vulgaris. BMJ. 2008 Jul 9;337:a209.

18. Laskaris G, Sklavounou A, Stratigos J. Bullous pemphigoid, cicatricial pemphigoid and pemphigus vulgaris. A comparative clinical survey of 278 cases. Oral Surg Oral Med Oral Pathol 1982;54:656-62.

19. Lamey PJ, Rees TD, Binnie WH, Wright JM, Rankin KV, Simpson NB. Oral presentation of pemphigus vulgaris and its response to systemic steroid therapy. Oral Surg Oral Med Oral Pathol 1992;74:54-7.

20. Rabinowitz LG, Esterly NB. Inflammatory bullous diseases in children. Dermatol Clin 1993;11:56581.

21. Robinson JC, Lozada-Nur F, Frieden I. Oral pemphigus vulgaris: a review of the literature and a report on the management of 12 cases. Oral Surg Oral Med Oral Pathol Oral Radiol Endod 1997;84:349-55.

22. Scully C, Mignogna M. Oral mucosal disease: pemphigus. Oral mucosal disease: pemphigus. Br J Oral Maxillofac Surg. 2008 Jun;46(4):2727. Epub 2007 Sep 17.

23. Scully C, Paes de Almeida O, Porter SR, Gilkes JJ. Pemphigus vulgaris: the manifestations and 
long-term management of 55 patients with oral lesions. BrJ Dermatol 1999;140:84-9.

24. Ahmed AR, Moy R. Death in pemphigus. J Am Acad Dermatol 1982;7: 221-8.

25. Parks MS, Teraski PI, Ahmed AR, Tinari JL. HLADRW4 in $91 \%$ of Jewish pemphigus vulgaris patients. Lancet 1979; 2: 441-442.

26. Bagán JV, Scully C. Medicina y Patología Oral. Valencia: Medicina Oral. 2006.

27. Black M, Mignogna MD, Scully C. Number II. Pemphigus vulgaris. Oral Dis. 2005 May;11(3): 119-30. Review.

28. Lin MS, Swartz SJ, López A, Ding X, Fairley JA, Díaz LA. T-lymphocytes from a subset of patients with pemphigus vulgaris respond to both desmoglein 3 and desmoglein 1, J Invest Dermatol 1997;109:734-7.

29. Martell P, Joly P. Pemphigus: autoimmune diseases of keratinocyte's adhesion molecules. Clinics in Dermatology 2001;19:662-74.

30. Hertl M, Veldman C. T-cellular autoimmunity against desmogleins in pemphigus, an autoantibody- mediated bullous disorder of the skin. Autoinmun Rev 2003;2:278-83

31. Amagai M. Pemphigus vulgaris and its active disease mouse model. Curr Dir Autoimmun. 2008; 10:167-81.

32. Baroni A, Lanza A, Cirillo N, Brunetti G, Ruocco E, Ruocco V. Vesicular and bullous disorders: pemphigus. Dermatol Clin. 2007 Oct;25(4):597603.

33. Dudelzak J, Lesher JL Jr, Abdelsayed RA. Pyogenic granuloma and pemphigus vulgaris: An unusual association. J Am Acad Dermatol. 2008 Feb;58(2 Suppl):S52-3,

34. Singh M, Demetriades N, Papas A, Gagari E. A clinico-pathologic correlation. Pemphigus vulgaris. J Mass Dent Soc. 2008;56(4):42-4.
35. Murrell DF, Dick S, Ahmed AR, Amagai M, Barnadas MA, Borradori L, Bystryn JC, Cianchini G, Diaz L, Fivenson D, Hall R, Harman KE, Hashimoto T, Hertl M, Hunzelmann N, Iranzo P, Joly P, Jonkman MF, Kitajima Y, Korman NJ, Martin LK, Mimouni D, Pandya AG, Payne AS, Rubenstein D, Shimizu H, Sinha AA, Sirois D, Zillikens D, Werth VP. Consensus statement on definitions of disease, end points, and therapeutic response for pemphigus. J Am Acad Dermatol. 2008 Jun;58(6):1043-6. Epub 2008 Mar 14.

36. Milián MA, Jiménez Y. Enfermedades ampollares de la cavidad oral: pénfigo y penfigoide. RCOE. 1997;2:687-95.

37. Hernandez G, Arriba de La Fuente L, Lucas M. Enfermedades ampollosas de la mucosa bucal, características diferenciales clínicas, histológicas e inmunológicas. Med Oral 1999;4: 528-51.

38. Soames J, Southam JC, 1993, Oral pathology, 2nd edition. Oxford; Oxford Univ. Press.

39. Brenner S, Wolf R. Possible nutricional factors in induced pemphigus. Dermatology. 1994;189: 337-9.

40. Mutzsim DF, Pelc NJ, Anhalt GJ. Drug-induced pemphigus. Dermatol Clin. 1993;11:463-71. Mignona MD, Muzio L, Zeppa P, Ruocco V, Bucci E. Immunocytochemical detection of autoantibody deposits in Tzanck smears from patients with oral pemphigus. J Oral Pathol Med 1997;26:254-7.

41. Chrysomallis F, Ioannides D, Teknetzis A, Panagiotidou D, Minas A. Treatment of oral pemphigus vulgaris. Int J Dermatol 1994;33: 803-7.

42. Fine JD. Management of acquired bullous skin diseases. N Engl J Med 1995;333:1475-84.

43. Stanley JR. Therapy of pemphigus vulgaris. Arch Dermatol 1999;135:76-8. 
44. Mimouni D, Anhalt GJ. Pemphigus. Dermatologic therapy 2002; 15:362-8.

45. Mimouni D, Nousari CH, Cummis DL, Kouba DJ, David M, Anhalt GJ. Differences and similarities among expert opinions on the diagnosis and treatment of pemphigus vulgaris. J Am Acad Dermatol 2003;49:1059-62.

46. Hashimoto T. Treatment strategies for pemphigus vulgaris in Japan. Expert Opin Pharmacother. 2008 Jun;9(9):1519-30.

47. Prajapati V, Mydlarski PR. Advances in pemphigus therapy. Skin Therapy Lett. 2008 Apr;13(3):4-7.

48. Gonzalez-Moles MA, Morales P, Rodriguez-Archilla A, Ruiz-avila I, Gonzalez-Moles S. Treatment of severe chronic oral erosive lesions with clobetasol propionate in aqueous solution. Oral Surg Oral Med Oral Pathol Oral Radiol Endod 2002;93:264-70.

49. Jiménez-Soriano Y, Díaz-Fernández JM. Enfermedades ampollares en la cavidad oral: pénfigo. RCOE 2004;9(4):439-447.

50. Enk AH, Knop J. Mycophenolate is effective in the treatment of phemphigus vulgaris. Arch Dermathol 1999;135:54-6.

51. Camisa C, Meisler DM. Immunobullous diseases with ocular involvement. Dermatol Clin 1992; 10: 555-70.

52. Pasricha JS, Siddhartha S. Curative effect of dexamethasone-cyclophosphamide pulse therapy for the treatment of phemphigus vulgaris. Inter $\mathrm{J}$ Dermatol 1992;31:875-7.

53. Aoyama Y, Nagasawa C, Nagai M, Kitajima Y. Severe pemphigus vulgaris: successful combination therapy of plasmapheresis followed by intravenous high-dose immunoglobulin to prevent rebound increase in pathogenic IgG. Eur J Dermatol. 2008 Sep-Oct;18(5):557-60. Epub 2008 Aug 8.

54. Bystryn JC, Jiao D. IVIg selectively and rapidly decreases circulating pathogenic autoantibodies in pemphigus vulgaris. Autoimmunity. 2006 Nov;39(7):601-7.

55. Rosenkrantz WS. Pemphigus: current therapy. Vet Dermatol. 2004 Apr;15(2):90-8.

56. Lo Schiavo A, Sangiuliano S, Puca RV, Brunetti G, Ruocco E, Cozzi R. Pemphigus and chrysotherapy: all that glitters is not gold! Int J Dermatol. 2008 Jun;47(6):645-7.

57. Thomas I. Gold therapy and its indications in dermatology. A review. J Am Acad Dermatol. 1987 Apr;16(4):845-54. Review.

58. Allen CM, Camisa C. Paraneoplastic pemphigus: a review of the literature. Oral Dis. 2000 Jul;6 (4):208-14. Review.

59. Anhalt GJ. Paraneoplastic pemphigus. J Investigative Dermatology Symposium Proceedins 2004;9:29-33,

60. Zhu X, Zhang B. Paraneoplastic pemphigus. J Dermatol. 2007 Aug;34(8):503-11. Review.

61. Batista MD, Takano D, Lopes RD, Enokihara MM, Michalany NS, de Almeida FA. Paraneoplastic pemphigus associated with non-Hodgkin lymphoma. Paraneoplastic pemphigus associated with non-Hodgkin lymphoma. Dermatol Online J. 2008 Jun 15;14(6):11.

62. Camisa C, Helm TN. Paraneoplastic pemphigus is a distinct neoplasia-induced autoimmune disease. Arch Dermatol. 1993 Jul;129(7):883-6.

63. Helm TN, Camisa C, Valenzuela R, Allen CM. Paraneoplastic pemphigus. A distinct autoimmune vesiculobullous disorder associated with neoplasia. Oral Surg Oral Med Oral Pathol. 1993 Feb;75(2):209-13,

64. Kaplan I, Hodak E, Ackerman L, Mimouri D, Anhal GJ, Calderon S. Neoplasms associated with paraneoplastic pemphigus: a review with emphasis on non-hematologic malignancy and oral mucosal manifestations. Oral Oncology 2004;40:553-62. 
65. Wang J, Zhu X, Li R, Tu P, Wang R, Zhang L, Li T, Chen X, Wang A, Yang S, Wu Y, Yang H, Ji S. Paraneoplastic pemphigus associated with Castleman tumor: a commonly reported subtype of paraneoplastic pemphigus in China. Arch Dermatol. 2005 Oct;141(10):1285-93, Review.

66. Nunes RL, Moresco VR, Marley G, et al. Pénfigo vulgar. Caso clínico. Av. Odontoestomatol 2005; 21(4):189-93.
67. Bascones-Martínez A, Figuero Ruiz E, Esparzo Gómez G. Úlceras orales. Med Clin (Barc) 2005; 125(15):590-7.

\section{CORRESPONDENCIA}

Rita Matos-Cruz

C/ Irún, 7, 4 B

28008 Madrid. España

e-mail: ritamatoscruz@gmail.com 